\title{
Hyperkalemic periodic paralysis
}

INSERM

\section{Source}

INSERM. (1999). Orphanet: an online rare disease and orphan drug data base.

Hyperkalemic periodic paralysis. ORPHA:682

Hyperkalemic periodic paralysis (HyperPP) is a muscle disorder characterized by episodic attacks of muscle weakness associated with an increase in serum potassium concentration. 